\title{
Three-Dimensional Simulations of Offshore Oil Platform in Square and Diamond Arrangements
}

\author{
Saliha Nouri, ${ }^{1}$ Zouhair Hafsia, ${ }^{1}$ Salah Mahmoud Boulaaras $\mathbb{D}^{2,3}$ Ali Allahem ${ }^{2},{ }^{4}$ \\ Salem Alkhalaf, ${ }^{5}$ and Aldo Munoz Vazquez ${ }^{6}$ \\ ${ }^{1}$ Department of Physics, College of Science and Arts at ArRass, Qassim University, Saudi Arabia \\ ${ }^{2}$ Department of Mathematics, College of Science and Arts at ArRass, Qassim University, Saudi Arabia \\ ${ }^{3}$ Laboratory of Fundamental and Applied Mathematics of Oran (LMFAO), University of Oran 1, Ahmed Benbella, Algeria \\ ${ }^{4}$ Department of Mathematics, College of Science, Qassim University, Saudi Arabia \\ ${ }^{5}$ Department of Computer Science, College of Science and Arts at ArRass, Qassim University, Saudi Arabia \\ ${ }^{6}$ College of Engineering, Texas A\&M University, 6200 Tres Lagos Blvd, Higher Education Center at McAllen, Texas 78504, USA
}

Correspondence should be addressed to Ali Allahem; aallahem@qu.edu.sa

Received 19 January 2021; Revised 15 February 2021; Accepted 19 February 2021; Published 2 March 2021

Academic Editor: F. Rabiei

Copyright (C) 2021 Saliha Nouri et al. This is an open access article distributed under the Creative Commons Attribution License, which permits unrestricted use, distribution, and reproduction in any medium, provided the original work is properly cited.

\begin{abstract}
The interaction of the solitary wave with an oil platform composed of four vertical circular cylinders is investigated for two attack angle of the solitary wave $\beta=0^{\circ}$ (square arrangement) and $\beta=45^{\circ}$ (diamond arrangement). The solitary wave is generated using an internal source line as proposed by Hafsia et al. (2009). This generation method is extended to three-dimensional wave flow and is integrated into the PHOENICS code. The volume of fluid approach is used to capture the free surface evolution. The present model is validated in the case of a solitary wave propagating on a flat bottom for $H / h=0.25$ where $H$ is the wave height and $h$ is the water depth. Compared to the analytical solution, the pseudowavelength and the wave crest are well reproduced. For a solitary wave interacting with square and diamond cylinders, the simulated results show that the maximum run-ups are well reproduced. For the diamond arrangements, the diffraction process seems to not affect the maximum run-ups, which approached the isolated cylinder. For the square arrangement, the shielding effect leads to a maximum wave force more pronounced for the upstream cylinder array.
\end{abstract}

\section{Introduction}

In the last decades, many researchers have focused on searching different wave structures of nonlinear partial differential equations. The interested readers can see [1-4]. The offshore oil platforms and the coastal bridges are composed of multiple cylinders disposed in different arrangements. When the wave run-up and the following wave forces exceed the expected values, the safety of these structures is compromised. The available analytical solutions in the literature are only valuable under some limiting assumptions. For this reason, experimental and numerical methods are adopted to solve this wave-platform interaction problem.
The interaction of a solitary wave (representing a real tsunami wave) with a single circular cylinder was studied experimentally by Yates and Wang (1994) in [5]. The effect of a single row of circular cylinders on the transmission and reflected coefficients is studied experimentally by Huang (2010) in [6]. The consequent results are that the wave force acting on a coastal structure protected by these cylinders can be reduced to about $60 \%$ for $S=1.2 \mathrm{D}$, where $S$ is the distance between the centers of adjacent cylinders, and $D$ is the cylinder diameter. An experimental study was conducted by Huang (2007) in [7] to measure the reflection and transmission coefficients in the case of single and twin rows of rectangular cylinders, and simplified analytical expressions of these 
coefficients are proposed. Wang et al. (2021) in [8] investigate experimentally, the back and front run-up and wave forces induced by solitary wave for different truncated vertical cylinders. Secondary peak for both the front run-up and wave forces are observed due to the return flow.

An alternative method to the experimental measurements of the free surface evolution is the use of a twodimensional (2-D) or three-dimensional (3-D) numerical method. The first one is based on the depth averaged Boussinesq equations and can be used for a long-term simulation. Lin and Man (2007) in [9] validate the Boussinesq model for one-dimensional and two-dimensional wave transformations. Mohapatra et al. (2020) in [10] studied the regular wave diffraction by a floating fixed vertical cylinder by two methods: using a CFD code and analytically based on a Boussinesq model. Among the various Boussinesq models existing in the literature, the nonlinear effects cannot well be reproduced (Zhao et al., 2007 in [11], Wang and Ren, 1999 in [12], and Liu et al., 2012 in [13]). Hence, a full threedimensional (3-D) Navier-Stokes model is required. In order to study the regular wave run-ups for a single and a group of vertical cylinders, numerical virtual wave probes were used by Cao and Wan (2017) in [14]. Frantzis et al. (2020) in [15] adopted a (3-D) numerical wave tank (NWT) to study the wave breaking induced by a single row of vertical cylinders for different ranges of cylinder diameter to depth ratios. The large eddy simulation (LES) model was used to reproduce the small scales of turbulence. Numerical results show that the effect of the cylinder diameter is more significant for larger values of solitary wave heights. Wang et al. (2018) in [16] conducted a series of laboratory experiments on the internal solitary wave (ISW) loads upon semisubmersible platforms in a density stratified fluid tank, and investigated the load components induced by different factors. The wave loads on a platform composed of $2 \times 2$ circular cylinders in side-by-side and tandem arrangements are numerically studied using the Reynolds-Averaged Navier-Stokes equations by Yang et al. (2015) in [17]. The desired monochromatic wave was generated by the prescribed velocity components at the inlet of the computational domain. Using a CFD code, Kamath et al. (2015) in [18] investigate the diffraction of sinusoidal wave by $3 \times 3$ square array cylinders placed in proximity and show that the wave force is highest when the distance between the cylinder center is less than half of the incident sinusoidal wave. The numerical results show that if $S>4 D$, there is no interaction between the platform cylinders. Xie et al. in [19] used a cut-cell method in a fully (3D) code to simulate solitary wave interaction with a vertical circular cylinder and a thin horizontal plate. Several computational fluid dynamics (CFD) implemented a cut-cell algorithm permitting to identify the contribution of a portion of a rectangular grid to the convective and diffusive fluxes.

The main task of the present study is to investigate the interaction of the solitary wave with one or four circular cylinders in a square or diamond arrangement using a (3-D) numerical wave tank (NWT). The proposed wave generation method is based on an internal mass source. The cut-cell implemented in the PHOENICS code is used to reproduce the circular cylinder shape.

\section{Mathematical Formulation}

2.1. Computational Domain. The position of the still water level $h$ and the location of the mass source line for solitary wave generation are shown in Figure 1(a). The direction of propagation of the solitary wave is the positive $x$-direction. In all simulated cases, the depth to cylinder radius ratio is taken: $h / a=1$, and the distance from the center of cylinders is $S=3 a$. For the square platform, there is one cylinder in each corner of the square as indicated in Figure 1(b). The two upstream cylinders are denoted 3 and 4 , and the two downstream cylinders are 1 and 2 . The square arrangement corresponding to the attack angle of solitary wave $\beta=0$. This angle is measured between the propagating direction and the symmetric line of the platform (the line parallel to the line joining 1 and 3). The diamond configuration of the platform is shown in Figure 1(c) and corresponds to $\beta=45^{\circ}$. The effective computational domain has a length of $L=55 a$. Two dissipative zones are added in each open boundary to avoid wave reflection having a length of $(25 a)$. The considered width is $(10 a),(13 a)$, and $(12.25 a)$, respectively, for a single cylinder, square platform, and diamond platform.

The overall grid and the grid around the oil platform are shown in Figure 2 for the diamond arrangement. Fine grids are adopted around the four cylinders, and coarse grids are imposed in the two dissipation regions. The cut-cell method is used to mesh the circular cylinders in the Cartesian coordinates system. Figure 2 (b) shows the details of the cut-cell grids around the four cylinders. The number of grid in $x, y$, and $z$ directions is, respectively $\mathrm{NX} \times \mathrm{NY} \times \mathrm{NZ}=220 \times 110$ $\times 102$ for the diamond arrangement and $220 \times 100 \times 102$ for the square arrangement. For these two arrangements, the time step is $\Delta t=0.01 \mathrm{~s}$.

2.2. Governing Transport Equations. The proposed (NWT) was based on the full three-dimensional (3-D) Navier-Stokes transport equations coupled to the volume of liquid (VOF) convective transport equation to reproduce the water wave interface. For unsteady flow and incompressible fluid, the mass and momentum conservation equations are written as:

(i) The mass conservation equation:

$$
\frac{\partial \rho}{\partial t}+\frac{\partial u_{i}}{\partial x_{i}}=0
$$

(ii) The momentum transport equation:

$$
\frac{\partial u_{i}}{\partial t}+u_{j} \frac{\partial u_{i}}{\partial x_{j}}=-\frac{1}{\rho} \frac{\partial p}{\partial x_{i}}+\frac{\partial}{\partial x_{j}}\left[v\left(\frac{\partial u_{i}}{\partial x_{j}}+\frac{\partial u_{j}}{\partial x_{i}}\right)\right]+g_{i}+s_{d, i},
$$

where $x_{i}$ is the Cartesian coordinates, $u_{i}$ is the velocity components, $\rho$ is the density of the mixture, $p$ is the pressure, $v$ is the kinematic viscosity of the mixture, $g$ is the 


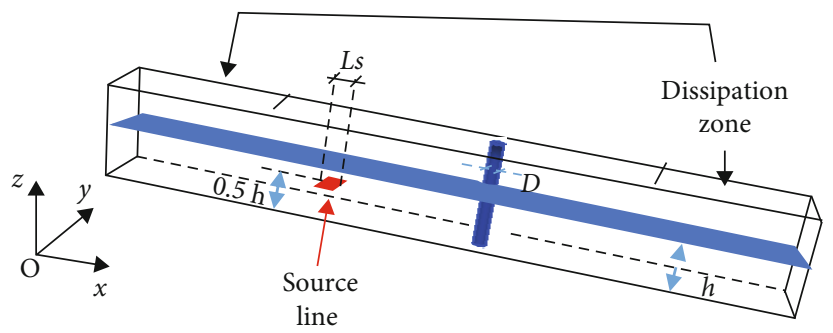

(a)

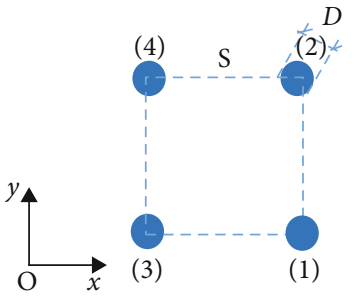

(b)

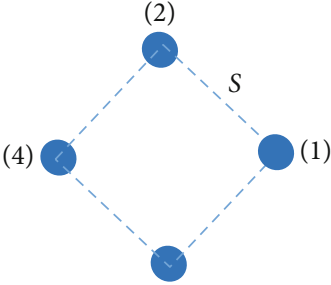

(3)

Figure 1: The computational domain and wave source line location; (a) side view; (b) top view.

acceleration due to gravity, and $s_{d, z}$ is a momentum source term added to the momentum equation along $z$-direction to avoid wave reflection at the open boundaries given by:

$$
s_{d, z}=\gamma(x) w
$$

where $\gamma(x)$ is a linear damping function and $w$ is the velocity component along the $z$-vertical direction.

2.3. Free Surface Capture. The air-water interface is modeled using the mixture model flow. If $\alpha_{q}$ denoted the volume fraction of the $q^{\text {th }}$ fluid in a cell, then,

(i) The density of the mixture is given by:

$$
\rho=\sum_{q=1}^{2} \rho_{q} \alpha_{q}
$$

where $\rho_{q}$ is the density of the water when $(q=1)$ and air when $(q=2)$.

(ii) And dynamic viscosity of the mixture is

$$
\mu=\sum_{q=1}^{2} \mu_{q} \alpha_{q}
$$

where $\mu_{q}$ is the dynamic viscosity of the water if $(q=1)$ and air when $(q=2)$.

The volume fraction of fluid is determined by the following mass conservation equation for each phase:

$$
\frac{\partial \alpha_{q}}{\partial t}+\frac{\partial\left(\alpha_{q} u_{i}\right)}{\partial x_{i}}=0 .
$$

When $\alpha_{q}=0$, the cell is occupied by air, $\alpha_{q}=1$, the cell is occupied by water, and $0<\alpha_{q}<1$, the cell contains the interface (Hirt and Nichols, 1981 in [20]).

2.4. Wave Generation. The desired solitary wave was generated by an internal source inlet across a source line as proposed by Hafsia et al. (2009) in [21]. The inlet vertical velocity is prescribed as a time-dependent inlet boundary condition:

$$
w^{I}=\frac{2 c \eta\left(x_{s}, t\right)}{L_{s}},
$$

where $L_{s}$ is the length of the internal source line. [22]):

The wave celerity is given by (Dominguez et al., 2019 in

$$
c=\sqrt{g(H+h)} .
$$

The solitary wave surface elevation $\eta\left(x_{s}, t\right)$ is given by the following equation:

$$
\eta=H \operatorname{sech}^{2}\left[k\left(x_{s}-c t\right)\right],
$$

where $H$ is the incident wave height and $t$ is the time. The distance $x_{s}$ permitting to have a negligible source at $t=0 \mathrm{~s}$ is determined by the following equation:

$$
x_{s}=\frac{4 h}{\sqrt{H / h}} .
$$

The equivalent wave number $k$ is

$$
k=\sqrt{\frac{3 H}{4 h^{2}(H+h)}} .
$$




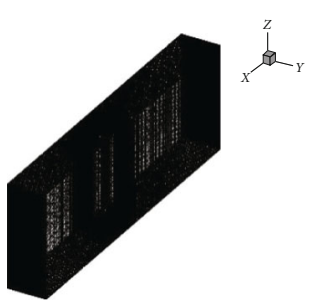

(a)

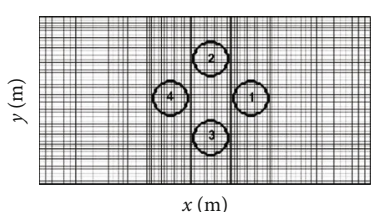

(b)

FIGURE 2: The grid of computational domain for the diamond arrangement of the four circular cylinders; (a) side view; (b) top view of the grid details around the cylinders by cut-cell method.

Following this equivalent wave number, the pseudowavelength can be determined by:

$$
L=\frac{2 \pi}{k}
$$

The length $L_{s}$ and the position of the source line are determined by the calibration procedure as indicated by Hafsia et al. (2009) in [21].

2.5. Wave Force. The wave force $\vec{F}$ acting on the cylinder is computed by integrating the water pressure $p$ and the normal component of the viscous stress tensor $\tau$ on the wetted surface of the cylinder $S$ :

$$
\vec{F}=\int_{S}(-\vec{n} p+\vec{n} \cdot \tau) d S
$$

where $\vec{n}$ is the normal unit vector pointing into the water.

From the component of this wave force along the $x$ -direction, the force coefficient can be calculated as:

$$
C_{f, x}=\frac{F_{x}}{\rho g h^{2} a} .
$$

2.6. Initial and Boundary Conditions. The following initial and boundary conditions are adopted for the governing transport equations. The imposed initial condition is still water with a depth $h$. For the top boundary, the pressure $P$ is set equal to the atmospheric pressure. Two dissipation zones are adopted at the open boundaries (Figure 1). At all the other boundaries of the computational domain, symmetric boundary conditions are imposed.

2.7. Numerical Schemes. To solve this proposed model, we adopt the PHOENICS code (Parabolic Hyperbolic or Elliptic Numerical Integration Code Series). In this code, the SIMPLEST iterative algorithm is used to solve the pressure and velocity coupling in the Navier-Stokes equations (Artemov et al., 2009 in [23]). The upwind scheme is used for nonlinear convection terms and an implicit formulation for the transient term. The (VOF) method is used to predict the interface between the water wave and air. For all the presented simulation results, the cut-cell within the PARSOL (PARtial SOLid) treatment detects the solid-fluid interface, which is not aligned with the Cartesian grid. The proposed three-

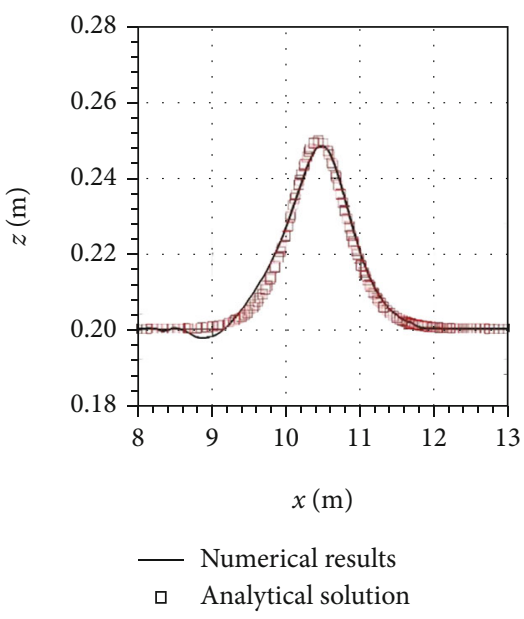

Figure 3: Comparison between the numerical and analytical free surface profiles at the centerline of the computational domain for $H / h=0.25$.

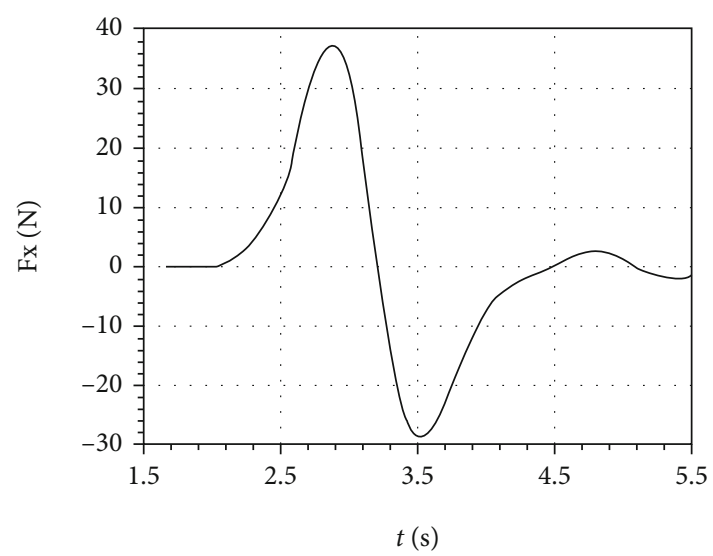

Figure 4: Time evolution of the in-line force on a single cylinder.

dimensional wave generation method is implemented in the PHOENICS code.

\section{Numerical Results}

3.1. Solitary Wave on a Single Cylinder. The proposed wave generation method based on an internal source line is validated for the nondimensional height $H / h=0.25$. The 


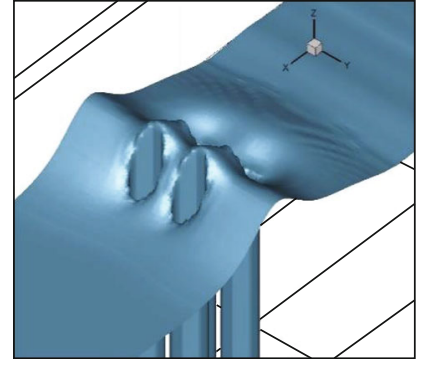

(a)

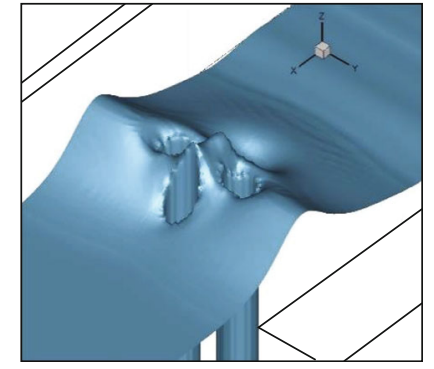

(b)

Figure 5: A three-dimensional free-surface elevation at the maximum run-up of the cylinder 1 for $H / h=0.25$ : (a) square arrangement; (b) diamond arrangement.

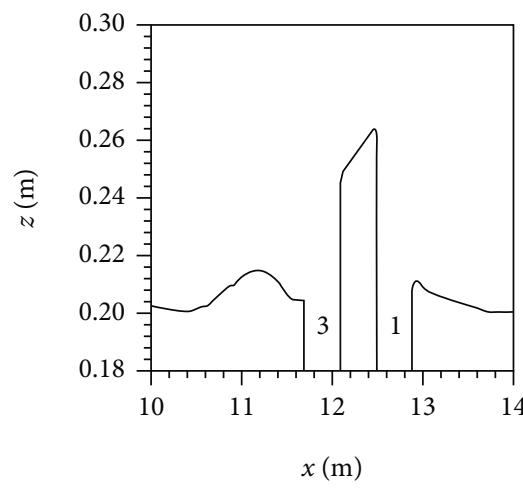

(a)

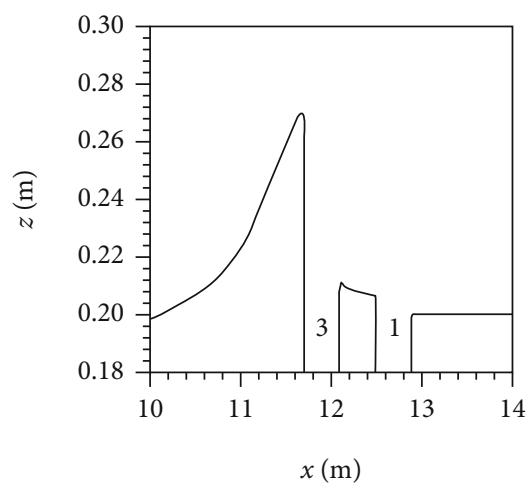

(c)

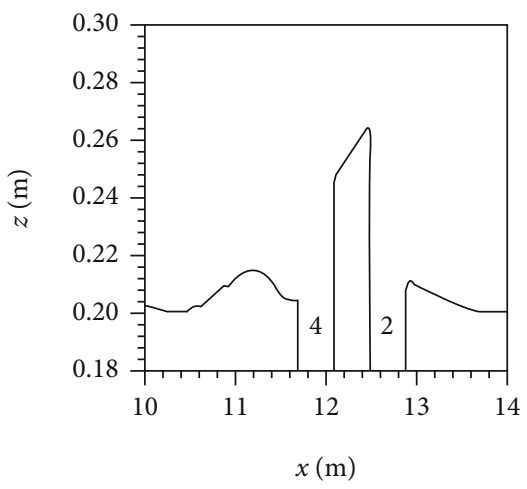

(b)

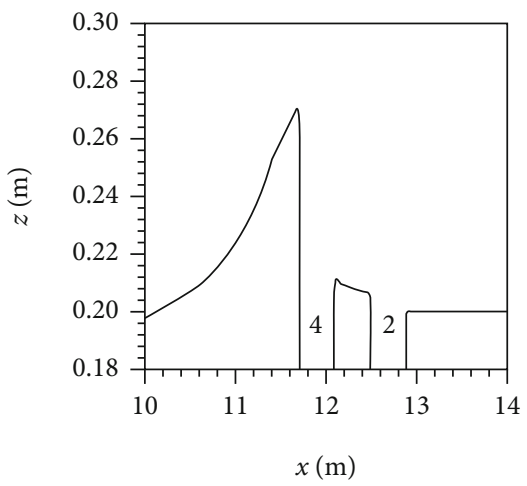

(d)

Figure 6: The maximum solitary wave run-ups at each cylinder for the square arrangement; (a) cylinder 1; (b) cylinder 2; (c) cylinder 3; (d) cylinder 4 .

simulated results show that before reaching the vertical cylinder, the wave profile is invariant in the transverse direction and can be represented by two-dimensional profiles. Figure 3 represents the simulated wave profiles at the center of the computational domain before impacting the cylinder. The wave is not affected by the cylinder, and the free surface profiles agree very well with the analytical one. The wave crest and the pseudowavelength are in accordance with the analytical one.

When a solitary wave passes around the cylinder, run-up occurs at the front of the cylinder and the maximum run-up depended on the incident wave energy. Then, the water level drops at the front producing the rise of the water level at the
TABle 1: Comparison of the simulated maximum run-up to the wave height ratio with Zhao et al. (2007) in [11] for a diamond arrangement.

\begin{tabular}{lcccc}
\hline & Cylinder 1 & Cylinder 2 & Cylinder 3 & Cylinder 4 \\
\hline $\begin{array}{l}\text { Present study } \\
\begin{array}{l}\text { Zhao et al. (2007) } \\
\text { in [11] }\end{array}\end{array}$ & 1.36 & 1.38 & 1.38 & 1.36 \\
\hline
\end{tabular}

rear of the cylinder by wave diffraction. In order to validate the cut-cell method, the maximum run-up is compared to the available literature. The maximum wave run-up is 


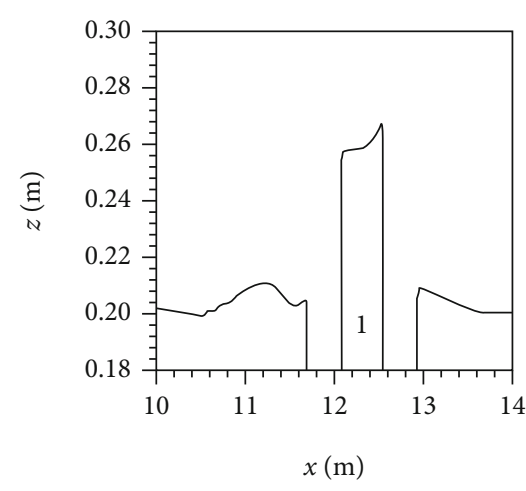

(a)

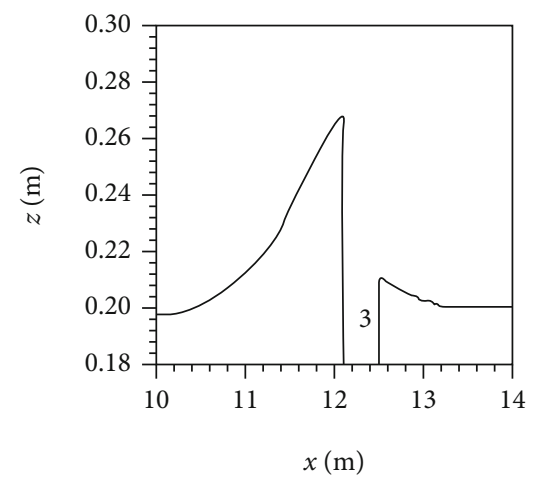

(c)

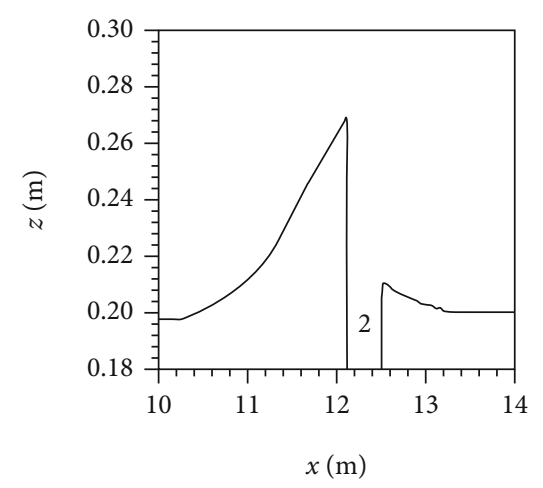

(b)

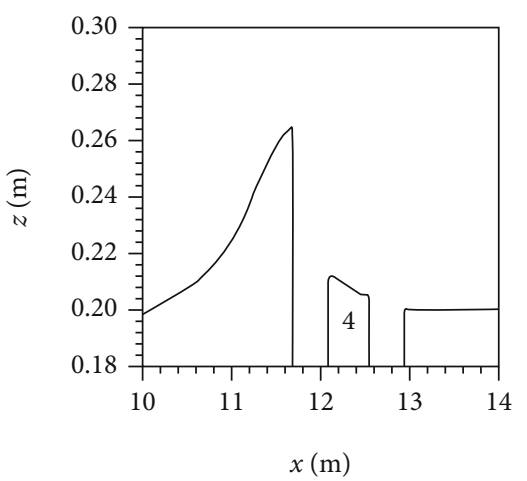

(d)

FIGURE 7: The maximum solitary wave run-ups at each cylinder for the diamond arrangement; (a) cylinder 1; (b) cylinder 2; (c) cylinder 3; (d) cylinder 4 .

determined from the evolution of the solitary wave profiles along the centerline and is equal to $R_{\max }=0.068 \mathrm{~m}$ corresponding to the ratio $R_{\max } / H=1.36$. Following the numerical study of Zhao et al. (2007) in [11] for the same $H / h=0.25$ and $h / a=1$, this ratio is found equal to 1.37 . There is no significant difference between this nondimensional run-up. The maximum wave force occurs at the same time as the maximum run-up that is equal to $t=2.9 \mathrm{~s}$. Figure 4 shows the time histories of the computed wave force on the $x$-direction acting on an isolated cylinder. The maximum wave force at this instant is equal to $F_{\max }=37.5 \mathrm{~N}$ corresponding to the following force coefficient $C_{f, x}=0.479$. The isolated cylinder is taken as a reference case for the computed force coefficient acting on each cylinder of the platform in the two studied configurations.

3.2. Solitary Wave Diffraction by an Oil Platform. The flow field due to the solitary wave diffraction by square and diamond platform is analyzed at the instant of the maximum run-up in terms of the free surface elevation, the maximum run-up $R_{\max }$ at each cylinder, and the maximum wave force $F_{\max }$.

The perspective view of the free surface elevation is shown in Figure 5 at the instant of the maximum run-up at cylinder 1 . The solitary wave crest has been altered by the diffraction process. Impacting the cylinder obstacle, the wave run-up is observed due to the transformation of the incident wave to potential energy. The $R_{\max }$ depends on the incoming
TABLE 2: Comparison of the simulated maximum run-up to the wave height ratio with Zhao et al. (2007) for a square arrangement.

\begin{tabular}{lcccc}
\hline & Cylinder 1 & Cylinder 2 & Cylinder 3 & Cylinder 4 \\
\hline $\begin{array}{l}\text { Present study } \\
\text { Zhao et al. (2007) }\end{array}$ & 1.29 & 1.28 & 1.42 & 1.42 \\
in [11] & 1.37 & 1.37 & 1.40 & 1.40 \\
\hline
\end{tabular}

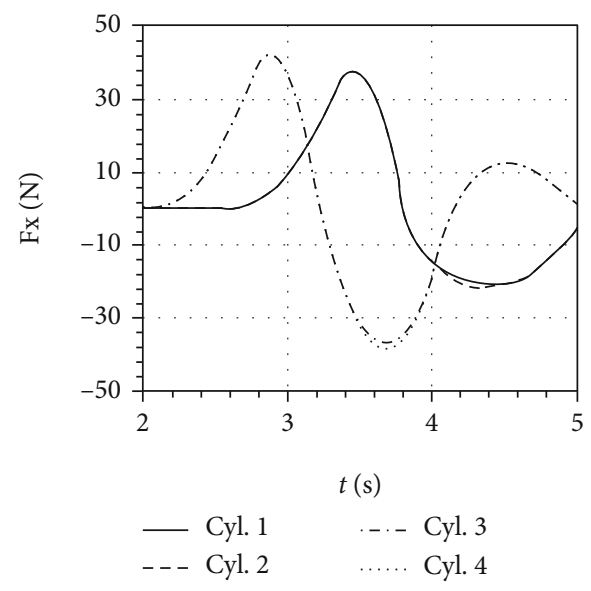

Figure 8: Time evolution of the in-line force on the square arrangement for $H / h=0.25$. 


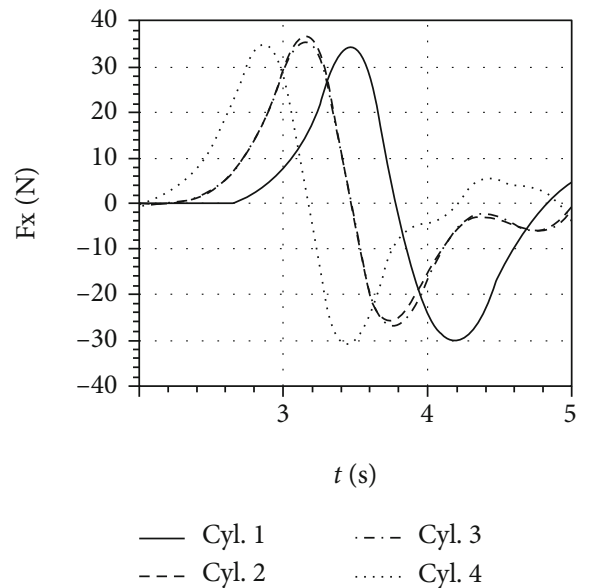

Figure 9: Time evolution of the in-line force on the diamond arrangement for $H / h=0.25$.

wave and the nature of the obstacle. This interaction leads to a complicated flow field as shown in Figure 5.

For square arrangement, the $R_{\max }$ for the first array (cylinders 3 and 4 ) occurs at the instant $t=2.90$ s and the for second array (cylinders 1 and 2) at $t=3.45 \mathrm{~s}$. For diamond arrangement, the first $R_{\max }$ is observed for the most upstream cylinder (cylinder 4 ) at $t=2.88 \mathrm{~s}$. The maximum run-ups for cylinders 2 and 3 occur at the same instant $(t=3.16 \mathrm{~s})$ and due to nonlinear effect, the $R_{\max }$ for cylinder 1 is observed at $t=3.48 \mathrm{~s}$.

Figure 6 represents the free surface elevation at the instant of the maximum run-up $R_{\max }$ for each cylinder in the square arrangement (zero solitary wave incidence). The $R_{\max }$ on the downstream cylinders (1 and 2) is smaller than those on the upstream cylinders ( 3 and 4 ). The $R_{\max }$ on cylinders 1 and 2 is smaller than on the corresponding isolated cylinder. This can be explained by the fact that some of the incident wave energy has been reflected back by other cylinders before the solitary wave impacting the downstream cylinders array. The numerical results of Zhao et al. (2007) in [11] confirm these conclusions, and the calculated $R_{\max } / H$ is closely the same as shown by Table 1 .

The free surface elevation at the instant of the maximum run-up $R_{\max }$ for each cylinder in the diamond arrangement is shown in Figure 7 . The $R_{\max } / H$ for cylinders 4 and 1 is located at the centerline approach to that on the isolated cylinder. The $R_{\max } / H$ for cylinders 2 and 3 is slightly greater than on the isolated cylinder. Table 2 shows good agreement between the present simulations and Zhao et al. in [11] results for the diamond arrangement.

The time evolution of the wave force in the positive $x-$ direction for each cylinder is presented in Figure 8 for the square arrangement. The maximum wave force $F_{\max }$ on the most downstream array of the cylinders ( 1 and 2$)$ is slightly greater than on the isolated cylinder. The increase of the $F_{\max }$ relative to the isolated cylinder is more pronounced for the first cylinder array (cylinders 3 and 4). The platform and wave interactions lead to $F_{\max }$ for the first cylinders (3 and 4) array greater than on the second array (cylinders 1 and 2). This can be attributed to the shielding effect of the upstream cylinder array. These conclusions are in concordance with the computed run-ups previously discussed.

For the diamond arrangement, Figure 9 presents the time evolution of the wave force for each cylinder. The aligned cylinders 4 and 1 have the same $F_{\max }$. Compared to the isolated cylinder, this $F_{\max }$ is significantly smaller. The two symmetric cylinders about the centerline of the computational domain are having the same $F_{\max }$ as the isolated cylinder. The approaching solitary wave for these cylinders seems to be not disturbed by the diffraction process.

\section{Conclusions}

A full three-dimensional numerical wave tank (NWT) was integrated on the PHOENICS code in order to study the solitary wave diffraction with diamond or square cylinders arrangements. The solitary wave was generated by an internal line source, and the cylinder structures are discretized using the cut-cell method. For the diamond platform arrangement, the maximum wave run-ups approach to that on the isolated cylinder indicating that the diffraction process does not affect the four cylinders. However, for a square arrangement, the shielding effect of the upstream cylinders leads to a maximum wave force for the first cylinders array greater than the most downstream array.

The cut-cell method can be generalized for more complex geometric coastal structures in the Cartesian coordinates system. The proposed model based on an internal line source for wave generation can be used to study the combined effects of wave and current on the forces acting on multiple cylinders. Different incident waves can be tested (monochromatic, Stokes, and cnoidal waves). The present model can be used to test the effect of the turbulence model, the effect of the wave height, and the distance between the cylinders on the wave forces and wave run-ups. Further wave-structure interaction cases can be studied such as the wave diffraction with floating structures.

\section{Data Availability}

No data were used to support the study.

\section{Conflicts of Interest}

This work does not have any conflicts of interest.

\section{Acknowledgments}

The third author would like to thank all the professors of the Mathematics Department at the University of Annaba in Algeria, especially his professors/scientists Pr. Mohamed Haiour, Pr. Ahmed-Salah Chibi, and Pr. Azzedine Benchettah for the important content of masters and $\mathrm{PhD}$ courses in pure and applied mathematics that he received during his studies. Moreover, he thanks them for the additional help they provided to him during office hours in their office about the few concepts/difficulties he had encountered, and he appreciates their talent and dedication for their postgraduate 
students currently and previously. The authors gratefully acknowledge Qassim University, represented by the Deanship of Scientific Research, on the financial support for this research under the number (10261-cos-2020-1-3-I) during the academic year $1442 \mathrm{AH} / 2020 \mathrm{AD}$.

\section{References}

[1] K. Hosseini, M. Samavat, M. Mirzazadeh, W. X. Ma, and Z. Hammouch, "A new $(3+1)$-dimensional Hirota bilinear equation: its Bäcklund transformation and rational-type solutions," Regular and Chaotic Dynamics, vol. 25, no. 4, pp. 383391, 2020.

[2] K. Hosseini, A. Seadawy, M. Mirzazadeh, M. Eslami, S. Radmehr, and D. Baleanu, "Multiwave, multicomplexiton, and positive multicomplexiton solutions to a $(3+1)$-dimensional generalized breaking soliton equation," Alexandria Engineering Journal, vol. 59, no. 5, pp. 3473-3479, 2020.

[3] K. Hosseini, W. A. Ma, R. Ansari, M. Mirzazadeh, M. Pouyanmehr, and F. Samadani, "Evolutionary behavior of rational wave solutions to the $(4+1)$-dimensional BoitiLeon-Manna-Pempinelli equation," Physica Scripta, vol. 95, article 065208, 2020.

[4] H. Hafsia, S. Nouri, S. Boulaaras, A. Allahem, A. Alkhalaf, and A. M. Vazquez, "Solitary wave diffraction with a single and two vertical circular cylinders," Mathematical Problems in Engineering, vol. 2021, Article ID 6634762, 9 pages, 2021.

[5] G. T. Yates and K. H. Wang, "Solitary wave scattering by a vertical cylinder: experimental study," in The Fourth International Offshore and Polar Engineering Conference, Osaka, Japan, 1994.

[6] Z. Huang, "Wave interaction with one or two rows of closely spaced rectangular cylinders," Ocean Engineering, vol. 34, no. 11-12, pp. 1584-1591, 2007.

[7] Z. Huang and Z. Yuan, "Transmission of solitary waves through slotted barriers: a laboratory study with analysis by a long wave approximation," Journal of Hydro-environment Research, vol. 3, pp. 179-185, 2010.

[8] Q. Wang, Y. Fang, and H. Liu, “An experimental study of runup and loads on a vertical truncated cylinder in a solitary wave," Ocean Engineering, vol. 219, pp. 1-10, 2021.

[9] P. Lin and C. Man, "A staggered-grid numerical algorithm for the extended Boussinesq equations," Applied Mathematical Modelling, vol. 31, no. 2, pp. 349-368, 2007.

[10] S. C. Mohapatra, H. Islam, and C. Guedes Soares, "Boussinesq model and CFD simulations of non-linear wave diffraction by a floating vertical cylinder," Journal of Marine Science and Engineering, vol. 8, no. 8, pp. 1-27, 2020.

[11] M. Zhao, L. Cheng, and B. Teng, "Numerical simulation of solitary wave scattering by a circular cylinder array," Ocean Engineering, vol. 34, no. 3-4, pp. 489-499, 2007.

[12] K.-H. Wang and X. Ren, "Interactions of cnoidal waves with cylinder arrays," Ocean Engineering, vol. 26, pp. 1-20, 1999.

[13] S. Liu, Z. Sun, and J. Li, "An unstructured FEM model based on Boussinesq equations and its application to the calculation of multidirectional wave run-up in a cylinder group," Applied Mathematical Modelling, vol. 36, no. 9, pp. 4146-4164, 2012.

[14] H. Cao and D. Wan, "Benchmark computations of wave runup on single cylinder and four cylinders by naoe-FOAMSJTU solver," Applied Ocean Research, vol. 65, pp. 327-337, 2017.
[15] C. Frantzis, D. G. E. Grigoriadis, and A. A. Dimas, "Numerical study of solitary waves past slotted breakwaters with a single row of vertical piles: wave processes and flow behavior," Ocean Engineering, vol. 211, no. 1, pp. 1-21, 2020.

[16] X. Wang, J. F. Zhou, Z. Wang, and Y. X. You, “A numerical and experimental study of internal solitary wave loads on semi-submersible platforms," Ocean Engineering, vol. 150, pp. 298-308, 2018.

[17] C. Yang, Y. Liu, and C. Liu, "Predicting wave loads on adjacent cylinder arrays with a 3D model," Journal of Hydraulic Research, vol. 53, no. 6, pp. 797-807, 2015.

[18] A. Kamath, M. Alagan Chella, H. Bihs, and Ø. A. Arntsen, "Evaluating wave forces on groups of three and nine cylinders using a 3D numerical wave tank," Engineering Applications of Computational Fluid Mechanics, vol. 9, no. 1, 2015.

[19] Z. Xie, T. Stoesser, S. Yan, Q. Ma, and P. Lin, "A Cartesian cutcell based multiphase flow model for large-eddy simulation of three-dimensional wave-structure interaction," Computers \& Fluids, vol. 213, no. 15, pp. 1-17, 2020.

[20] C. W. Hirt and B. D. Nichols, "Volume of fluid (VOF) method for the dynamics of free boundaries," Journal of Computational Physics, vol. 39, no. 1, pp. 201-225, 1981.

[21] Z. Hafsia, M. B. Hadj, H. Lamloumi, and K. Maalel, "Internal inlet for wave generation and absorption treatment," Coastal Engineering, vol. 56, no. 9, pp. 951-959, 2009.

[22] J. M. Domínguez, C. Altomare, J. Gonzalez-Caoa, and P. Lomonaco, "Towards a more complete tool for coastal engineering: solitary wave generation, propagation and breaking in an SPH-based model," Coastal Engineering Journal, vol. 61, pp. 1-15, 2019.

[23] V. Artemov, S. B. Beale, G. de Vahl Davis et al., "A tribute to DB Spalding and his contributions in science and engineering," International Journal of Heat and Mass Transfer, vol. 52, no. 17-18, pp. 3884-3905, 2009. 